\title{
Stolarsky-Puebla index
}

\author{
J. A. Méndez-Bermúdez ${ }^{1, *}$, R. Aguilar-Sánchez ${ }^{2}$, Ricardo Abreu Blaya ${ }^{3}$, José M. Sigarreta $^{3}$ \\ ${ }^{1}$ Instituto de Física, Benemérita Universidad Autónoma de Puebla, Apartado Postal J-48, Puebla 72570, Mexico \\ ${ }^{2}$ Facultad de Ciencias Químicas, Benemérita Universidad Autónoma de Puebla, Puebla 72570, Mexico \\ ${ }^{3}$ Facultad de Matemáticas, Universidad Autónoma de Guerrero, Carlos E. Adame No.54 Col. Garita, Acapulco Gro. 39650 , Mexico
}

(Received: 9 September 2021. Accepted: 20 September 2021. Published online: 27 September 2021.)

(C) 2021 the authors. This is an open access article under the CC BY (International 4.0) license (www.creativecommons.org/licenses/by/4.0/).

\begin{abstract}
We introduce a degree-based variable topological index inspired on the Stolarsky mean (known as the generalization of the logarithmic mean). We name this new index as the Stolarsky-Puebla index: $S P_{\alpha}(G)=\sum_{u v \in E(G)} d_{u}$, if $d_{u}=d_{v}$, and $S P_{\alpha}(G)=\sum_{u v \in E(G)}\left[\left(d_{u}^{\alpha}-d_{v}^{\alpha}\right) /\left(\alpha\left(d_{u}-d_{v}\right)\right]^{1 /(\alpha-1)}\right.$, otherwise. Here, uv denotes the edge of the network $G$ connecting the vertices $u$ and $v, d_{u}$ is the degree of the vertex $u$, and $\alpha \in \mathbb{R} \backslash\{0,1\}$. We also consider the limiting cases $S P_{\alpha \rightarrow 0}(G)$ and $S P_{\alpha \rightarrow 1}(G)$ that we name as the logarithmic-mean index and the identric-mean index, respectively. Indeed, for given values of $\alpha$, the Stolarsky-Puebla index reproduces well-known topological indices such as the reciprocal Randic index, the first Zagreb index, and several mean Sombor indices. Moreover, we apply these indices to random networks and demonstrate that $\left\langle S P_{\alpha}(G)\right\rangle$, normalized to the order of the network, scale with the corresponding average degree $\langle d\rangle$. Some mathematical properties of the Stolarsky-Puebla index are also discussed.
\end{abstract}

Keywords: degree-based topological index; Stolarsky mean; random networks.

2020 Mathematics Subject Classification: 05C50, 05C80, 05C92, 60B20.

\section{Introduction}

For two positive real numbers $x, y$, the Stolarsky mean $S_{\alpha}(x, y)$ is defined as [22]

$$
S_{\alpha}(x, y)=\lim _{(\xi, \eta) \rightarrow(x, y)}\left(\frac{\xi^{\alpha}-\eta^{\alpha}}{\alpha(\xi-\eta)}\right)^{1 /(\alpha-1)}= \begin{cases}x & \text { if } x=y, \\ \left(\frac{x^{\alpha}-y^{\alpha}}{\alpha(x-y)}\right)^{1 /(\alpha-1)} & \text { otherwise },\end{cases}
$$

here, $\alpha \in \mathbb{R} \backslash\{0,1\}$. In fact, $S_{\alpha}(x, y)$ is known as the generalization of the logarithmic mean [16]

$$
\operatorname{LogMean}(x, y)= \begin{cases}x & \text { if } x=y \\ \frac{x-y}{\ln x-\ln y} & \text { otherwise. }\end{cases}
$$

For given values of $\alpha, S_{\alpha}(x, y)$ reproduces known means including the logarithmic mean, when $\alpha \rightarrow 0$, and some cases of the power mean $[5,23]$

$$
P M_{\alpha}(x, y)=\left(\frac{x^{\alpha}+y^{\alpha}}{2}\right)^{1 / \alpha} .
$$

As examples, in Table 1 we show some expressions for $S_{\alpha}(x, y)$ for selected values of $\alpha$ with their corresponding names, when available.

Also, there is a well-known inequality relating the Stolarsky mean and the power mean, namely $[6,16,19]$ :

$$
S_{-1}(x, y)=P M_{\alpha \rightarrow 0}(x, y) \leq S_{\alpha \rightarrow 0}(x, y) \leq P M_{1 / 3}(x, y) \leq S_{2}(x, y)=P M_{1}(x, y)
$$

or more explicitely

$$
\sqrt{x y} \leq \operatorname{LogMean}(x, y) \leq\left(\frac{x^{1 / 3}+y^{1 / 3}}{2}\right)^{3} \leq \frac{x+y}{2},
$$

where the equality is attained when $x=y$.

\footnotetext{
*Corresponding author (jmendezb@ifuap.buap.mx).
} 
Table 1: Expressions for the Stolarsky mean $S_{\alpha}(x, y)$ for selected values of $\alpha$.

\begin{tabular}{|c|c|c|}
\hline$\overline{\alpha \alpha}$ & 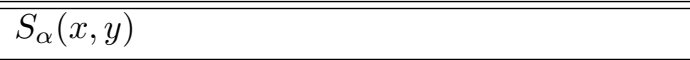 & " name (when available) \\
\hline$-\infty$ & $S_{\alpha \rightarrow-\infty}(x, y)=\min (x, y)$ & minimum value, $P M_{\alpha \rightarrow-\infty}(x, y)$ \\
\hline-4 & $S_{-4}(x, y)=\left(\frac{x^{3}+x^{2} y+x y^{2}+y^{3}}{4 x^{4} y^{4}}\right)^{-1 / 5}$ & \\
\hline-3 & $S_{-3}(x, y)=\left(\frac{x^{2}+x y+y^{2}}{3 x^{3} y^{3}}\right)^{-1 / 4}$ & \\
\hline-2 & $S_{-2}(x, y)=\left(\frac{x+y}{2 x^{2} y^{2}}\right)^{-1 / 3}$ & \\
\hline-1 & $S_{-1}(x, y)=\sqrt{x y}$ & geometric mean, $P M_{\alpha \rightarrow 0}(x, y)$ \\
\hline 0 & $S_{\alpha \rightarrow 0}(x, y)= \begin{cases}x & \text { if } x=y \\
\frac{x-y}{\ln x-\ln y} & \text { otherwise }\end{cases}$ & $\operatorname{LogMean}(x, y)$ \\
\hline $1 / 2$ & $S_{1 / 2}(x, y)=\left(\frac{\sqrt{x}+\sqrt{y}}{2}\right)^{2}$ & $P M_{1 / 2}(x, y)$ \\
\hline 1 & $S_{\alpha \rightarrow 1}(x, y)= \begin{cases}x & \text { if } x=y \\
\frac{x-y}{x \ln x-y \ln y} & \text { otherwise }\end{cases}$ & identric mean \\
\hline 2 & $S_{2}(x, y)=\frac{x+y}{2}$ & arithmetic mean, $P M_{1}(x, y)$ \\
\hline 3 & $S_{3}(x, y)=\left(\frac{x^{2}+x y+y^{2}}{3}\right)^{1 / 2}$ & \\
\hline 4 & $S_{4}(x, y)=\left(\frac{x^{3}+x^{2} y+x y^{2}+y^{3}}{4}\right)^{1 / 3}$ & \\
\hline$\infty$ & $S_{\alpha \rightarrow \infty}(x, y)=\max (x, y)$ & maximum value, $P M_{\alpha \rightarrow \infty}(x, y)$ \\
\hline
\end{tabular}

\section{Stolarsky-Puebla index}

A large number of graph invariants of the form

$$
T I(G)=\sum_{u v \in E(G)} F\left(d_{u}, d_{v}\right)
$$

are currently being studied in mathematical chemistry; where $u v$ denotes the edge of the graph $G$ connecting the vertices $u$ and $v, d_{u}$ is the degree of the vertex $u$, and $F(x, y)$ is an appropriate chosen function, see e.g. [12].

Inspired by the Stolarsky mean and given a simple graph $G=(V(G), E(G))$, here we choose the function $F(x, y)$ in (5) as the Stolarsky mean $S_{\alpha}(x, y)$ and define the degree-based variable topological index

$$
S P_{\alpha}(G)=\sum_{u v \in E(G)} S_{\alpha}\left(d_{u}, d_{v}\right)=\sum_{u v \in E(G)} \begin{cases}d_{u} & \text { if } d_{u}=d_{v} \\ \left(\frac{d_{u}^{\alpha}-d_{v}^{\alpha}}{\alpha\left(d_{u}-d_{v}\right)}\right)^{1 /(\alpha-1)} & \text { otherwise }\end{cases}
$$

where $u v$ denotes the edge of the graph $G$ connecting the vertices $u$ and $v, d_{u}$ is the degree of the vertex $u$, and $\alpha \in \mathbb{R} \backslash\{0,1\}$. We name $S P_{\alpha}(G)$ as the Stolarsky-Puebla index.

Note that for given values of $\alpha, S P_{\alpha}(G)$ is related to widely studied topological indices: $S P_{-1}(G)=R^{-1}(G)$, where $R^{-1}(G)$ is the reciprocal Randic index [13], $S P_{1 / 2}(G)=2^{-2} K A_{1 / 2,2}^{1}(G)$, where $K A_{\alpha, \beta}^{1}(G)$ is the first $(\alpha, \beta)-K A$ index [15], and $S P_{2}(G)=M_{1}(G) / 2$, where $M_{1}(G)$ is the first Zagreb index [14]. Also, for selected values of $\alpha, S P_{\alpha}(G)$ reproduces several mean Sombor indices

$$
m S O_{\alpha}(G)=\sum_{u v \in E(G)}\left(\frac{d_{u}^{\alpha}+d_{v}^{\alpha}}{2}\right)^{1 / \alpha}
$$

recently introduced in [2]. In Table 2, we report some expressions for $S P_{\alpha}(G)$ for selected values of $\alpha$ that we identify with known topological indices, when applicable. 
Table 2: Expressions for the Stolarsky-Puebla index $S P_{\alpha}(G)$ for selected values of $\alpha$.

\begin{tabular}{|c|c|c|}
\hline$\alpha$ & $S P_{\alpha}(G)$ & index equivalence \\
\hline$-\infty$ & $S P_{\alpha \rightarrow-\infty}(G)=\sum_{u v \in E(G)} \min \left(d_{u}, d_{v}\right)$ & $m S O_{\alpha \rightarrow-\infty}(G)$ \\
\hline-4 & $S P_{-4}(G)=\sum_{u v \in E(G)}\left(\frac{d_{u}^{3}+d_{u}^{2} d_{v}+d_{u} d_{v}^{2}+d_{v}^{3}}{4 d_{u}^{4} d_{v}^{4}}\right)^{-1 / 5}$ & \\
\hline-3 & $S P_{-3}(G)=\sum_{u v \in E(G)}\left(\frac{d_{u}^{2}+d_{u} d_{v}+d_{v}^{2}}{3 d_{u}^{3} d_{v}^{3}}\right)^{-1 / 4}$ & \\
\hline-2 & $S P_{-2}(G)=\sum_{u v \in E(G)}\left(\frac{d_{u}+d_{v}}{2 d_{u}^{2} d_{v}^{2}}\right)^{-1 / 3}$ & \\
\hline-1 & $S P_{-1}(G)=\sum_{u v \in E(G)} \sqrt{d_{u} d_{v}}$ & $R^{-1}(G)=m S O_{\alpha \rightarrow 0}(G)$ \\
\hline 0 & $S P_{\alpha \rightarrow 0}(G)=\sum_{u v \in E(G)} \begin{cases}d_{u} & \text { if } d_{u}=d_{v} \\
\frac{d_{u}-d_{v}}{\ln d_{u}-\ln d_{v}} & \text { otherwise }\end{cases}$ & logarithmic-mean index, see (18) \\
\hline $1 / 2$ & $S P_{1 / 2}(G)=\sum_{u v \in E(G)}\left(\frac{\sqrt{d_{u}}+\sqrt{d_{v}}}{2}\right)^{2}$ & $2^{-2} K A_{1 / 2,2}^{1}(G)=m S O_{1 / 2}(G)$ \\
\hline 1 & $S P_{\alpha \rightarrow 1}(G)=\sum_{u v \in E(G)} \begin{cases}d_{u} & \text { if } d_{u}=d_{v} \\
\frac{d_{u}-d_{v}}{d_{u} \ln d_{u}-d_{v} \ln d_{v}} & \text { otherwise }\end{cases}$ & identric-mean index, see (19) \\
\hline 2 & $S P_{2}(G)=\sum_{u v \in E(G)} \frac{d_{u}+d_{v}}{2}$ & $2^{-1} M_{1}(G)=m S O_{1}(G)$ \\
\hline 3 & $S P_{3}(G)=\sum_{u v \in E(G)}\left(\frac{d_{u}^{2}+d_{u} d_{v}+d_{v}^{2}}{3}\right)^{1 / 2}$ & \\
\hline 4 & $S P_{4}(G)=\sum_{u v \in E(G)}\left(\frac{d_{u}^{3}+d_{u}^{2} d_{v}+d_{u} d_{v}^{2}+d_{v}^{3}}{4}\right)^{1 / 3}$ & \\
\hline$\infty$ & $S P_{\alpha \rightarrow \infty}(G)=\sum_{u v \in E(G)} \max \left(d_{u}, d_{v}\right)$ & $m S O_{\alpha \rightarrow \infty}(G)$ \\
\hline
\end{tabular}

\section{Computational study of $S P_{\alpha}(G)$ on random networks}

As a first test of the Stolarsky-Puebla index, here we apply it on two models of random networks: Erdös-Rényi (ER) networks and random geometric (RG) graphs. ER networks [8-10,21] $G_{\mathrm{ER}}(n, p)$ are formed by $n$ vertices connected independently with probability $p \in[0,1]$. While RG graphs $[7,20] G_{\mathrm{RG}}(n, r)$ consist of $n$ vertices uniformly and independently distributed on the unit square, where two vertices are connected by an edge if their Euclidean distance is less or equal than the connection radius $r \in[0, \sqrt{2}]$.

We stress that the computational study of the Stolarsky-Puebla index we perform here is justified by the random nature of the network models we want to explore. Since a given parameter set $[(n, p)$ or $(n, r)]$ represents an infinite-size ensemble of random [ER or RG] networks, the computation of $S P_{\alpha}(G)$ on a single network is irrelevant. In contrast, the computation of the average value of $S P_{\alpha}(G)$ on a large ensemble of random networks, all characterized by the same parameter set, may provide useful average information about the full ensemble. This statistical approach, well known in random matrix theory studies, has been recently applied to random networks by means of topological indices, see e.g. [1,17,18]. Moreover, it has been shown that average topological indices may serve as complexity measures equivalent to standard random matrix theory measures $[3,4]$.

\section{1. $S P_{\alpha}(G)$ on Erdös-Rényi random networks}

In what follows, we present the average values of selected Stolarsky-Puebla indices. All averages are computed over ensembles of $10^{7} / n$ ER networks characterized by the parameter pair $(n, p)$.

In Figure 1, we present the average Stolarsky-Puebla index $\left\langle S P_{\alpha}\left(G_{\mathrm{ER}}\right)\right\rangle$ for $\alpha \rightarrow-\infty, \alpha \rightarrow 0, \alpha \rightarrow 1$, and $\alpha \rightarrow \infty$ as a 

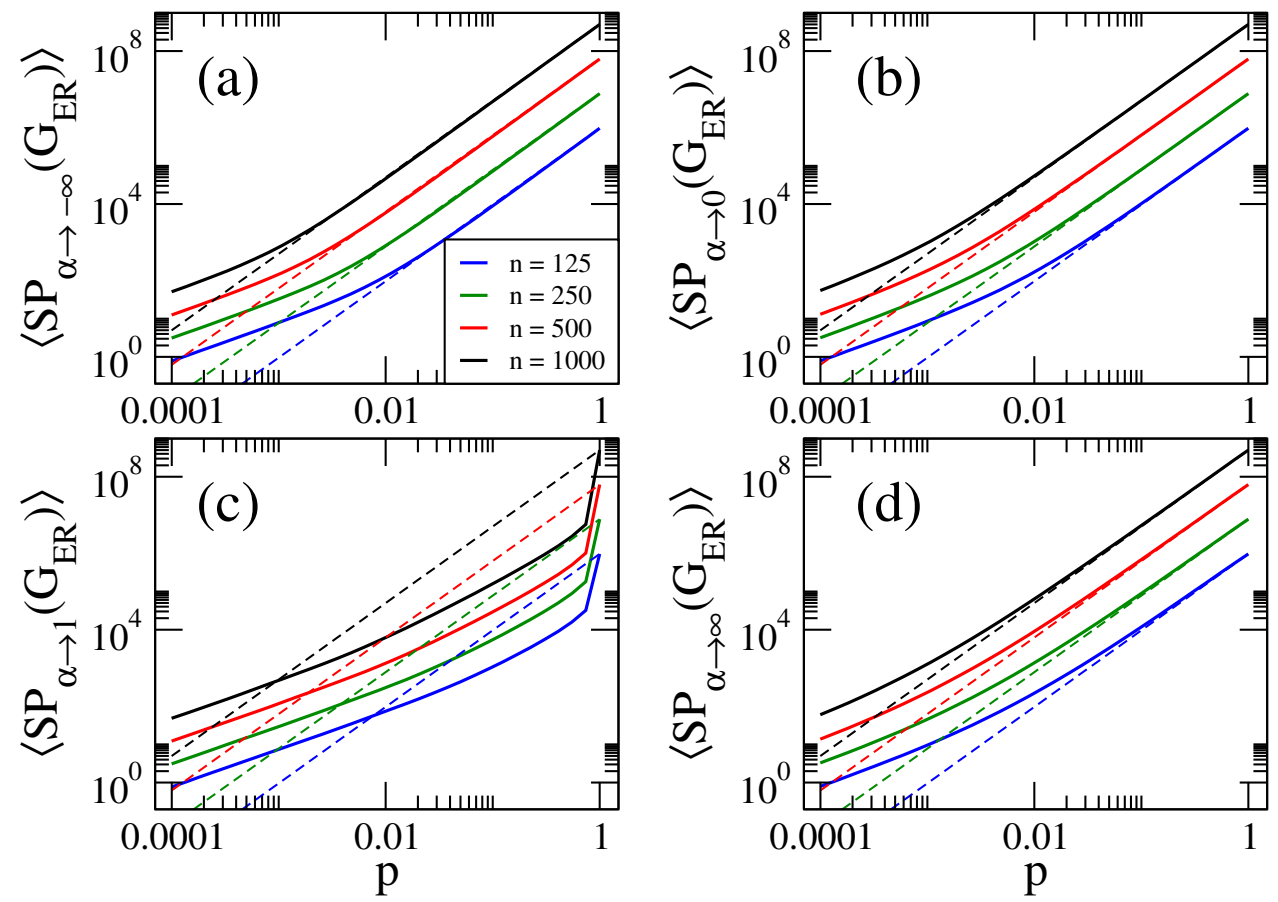

Figure 1: Average value of the Stolarsky-Puebla index $\left\langle S P_{\alpha}\left(G_{\mathrm{ER}}\right)\right\rangle$ as a function of the probability $p$ of Erdös-Rényi networks of size $n$. Here (a) $\alpha \rightarrow-\infty$, (b) $\alpha \rightarrow 0$, (c) $\alpha \rightarrow 1$, and (d) $\alpha \rightarrow \infty$. Dashed lines correspond to (9).

function of the probability $p$ of ER networks of sizes $n=\{125,250,500,1000\}$. From this figure we observe that the curves of $\left\langle S P_{\alpha}\left(G_{\mathrm{ER}}\right)\right\rangle$ are monotonically increasing functions of $p$.

We note that in the dense limit, i.e. when $n p \gg 1$, we can approximate $d_{u} \approx d_{v} \approx\langle d\rangle$ in (6), with

$$
\langle d\rangle=(n-1) p .
$$

Thus, when $n p \gg 1$, we can approximate $S P_{\alpha}\left(G_{\mathrm{ER}}\right)$ as

$$
S P_{\alpha}\left(G_{\mathrm{ER}}\right) \approx \sum_{u v \in E(G)} d_{u} \approx \sum_{u v \in E(G)}\langle d\rangle \approx \frac{1}{2} n[(n-1) p]^{2},
$$

where we have used $\left|E\left(G_{\mathrm{ER}}\right)\right|=n(n-1) p / 2$. In Figure 1, we show that (9) (dashed lines) indeed describes well the data (thick full curves) for large enough $p$; except for the case $\left\langle S P_{\alpha \rightarrow 1}\left(G_{\mathrm{ER}}\right)\right\rangle$, see Figure 1(c). We also verified that (9) describes well the data for other values of $\alpha$, however we did not include them in Figure 1 to avoid figure saturation. We also observed that the smaller the value of $\alpha$ the wider the range of $p$ where the coincidence between (9) and the computational data is observed; compare for example Figs. 1(a) and 1(d), where it is clear that the correspondence of the computational data with (9) is much better in the case of $\alpha \rightarrow-\infty$ than for $\alpha \rightarrow \infty$. In addition, it is relevant to note that (9) does not depend on $\alpha$.

We also notice that in Figure 1 we present average Stolarsky-Puebla indices as a function of the probability $p$ of ER networks of four different sizes $n$. It is quite clear from these figures that the curves, characterized by the different network sizes, are very similar but displaced on both axes. This behavior suggests that the average Stolarsky-Puebla indices can be scaled, as will be shown below.

From (9) we observe that $\left.\left\langle S P_{\alpha}\left(G_{\mathrm{ER}}\right)\right\rangle \propto n f[(n-1) p)\right]$ or

$$
\left\langle S P_{\alpha}\left(G_{\mathrm{ER}}\right)\right\rangle \propto n f(\langle d\rangle) .
$$

Therefore, in Figure 2 we plot again the average Stolarsky-Puebla indices reported in Figure 1, but now normalized to $n$, as a function of $\langle d\rangle$ showing that all indices are now properly scaled; i.e. the curves painted in different colors for different network sizes fall on top of each other. Moreover, we can rewrite (10) as

$$
\frac{\left\langle S P_{\alpha}\left(G_{\mathrm{ER}}\right)\right\rangle}{n} \approx \frac{1}{2}\langle d\rangle^{2} .
$$

In Figure 2, we show that (11) (orange-dashed lines) indeed describe well the computational data (thick full curves) for $\langle d\rangle \geq 10$; except for $\left\langle S P_{\alpha \rightarrow 1}\left(G_{\mathrm{ER}}\right)\right\rangle$, see Figure 2(c).

It is relevant to stress that even when (10) was expected to be valid in the dense limit (i.e. for $\langle d\rangle \gg 1$ ), it is indeed valid for any $\langle d\rangle$ as clearly seen in Figure 2. 


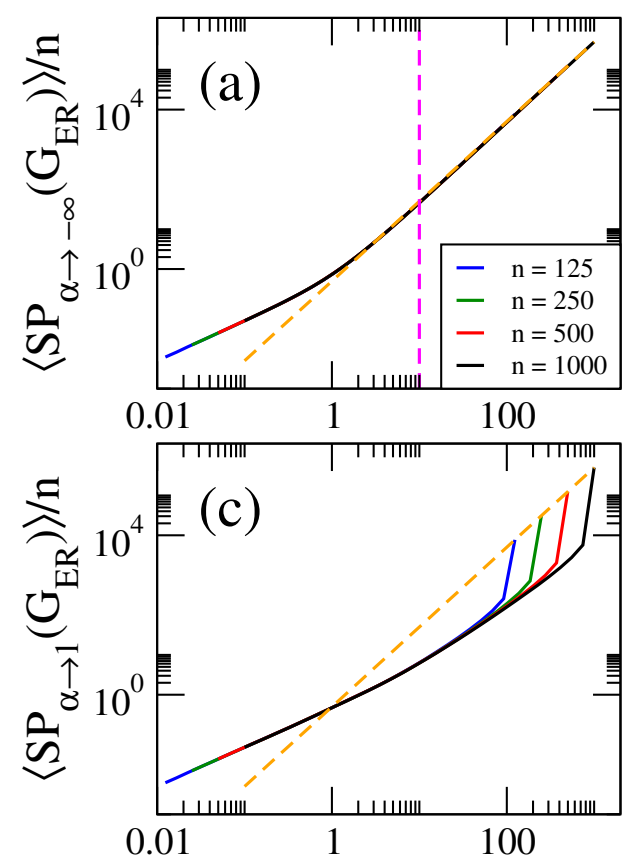

$\langle d\rangle$

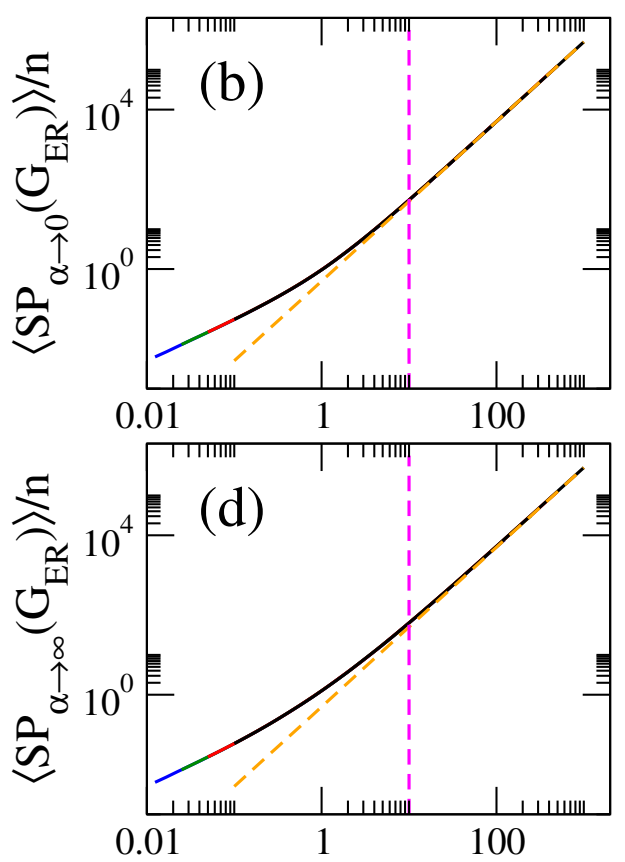

$\langle d\rangle$

Figure 2: Average value of the Stolarsky-Puebla index $\left\langle S P_{\alpha}\left(G_{\mathrm{ER}}\right)\right\rangle$, normalized to the network size $n$, as a function of the average degree $\langle d\rangle$ of Erdös-Rényi networks. Same curves as in Figure 1. Orange dashed lines are (11). The vertical magenta dashed lines indicate $\langle d\rangle=10$.

\section{2. $S P_{\alpha}(G)$ on random geometric graphs}

As in the previous Subsection, here we present the average values of selected Stolarsky-Puebla indices. Again, all averages are computed over ensembles of $10^{7} / n$ random graphs, each ensemble characterized by a fixed parameter pair $(n, r)$.

In Figure 3 we present the average Stolarsky-Puebla index $\left\langle S P_{\alpha}\left(G_{\mathrm{ER}}\right)\right\rangle$ for $\alpha \rightarrow-\infty, \alpha \rightarrow 0, \alpha \rightarrow 1$, and $\alpha \rightarrow \infty$ as a function of the connection radius $r$ of RG graphs of sizes $n=\{125,250,500,1000\}$. For comparison purposes, Figure 3 is equivalent to Figure 1. In fact, all the observations made in the previous Subsection for ER networks are also valid for RG graphs by just replacing $G_{\mathrm{ER}} \rightarrow G_{\mathrm{RG}}$ and $p \rightarrow g(r)$, with [11]

$$
g(r)= \begin{cases}r^{2}\left[\pi-\frac{8}{3} r+\frac{1}{2} r^{2}\right] & 0 \leq r \leq 1, \\ \frac{1}{3}-2 r^{2}[1-\arcsin (1 / r)+\arccos (1 / r)]+\frac{4}{3}\left(2 r^{2}+1\right) \sqrt{r^{2}-1}-\frac{1}{2} r^{4} & 1 \leq r \leq \sqrt{2} .\end{cases}
$$

As well as for ER networks, here, in the dense limit, when $n r \gg 1$, we can approximate $d_{u} \approx d_{v} \approx\langle d\rangle$ with

$$
\langle d\rangle=(n-1) g(r) .
$$

Therefore, in the dense limit, $S P_{\alpha}\left(G_{\mathrm{RG}}\right)$ is well approximated by:

$$
S P_{\alpha}\left(G_{\mathrm{RG}}\right) \approx \frac{1}{2} n[(n-1) g(r)]^{2} .
$$

In Figure 3, we show that (14) (dashed lines) indeed describes well the data (thick full curves) for large enough $r$; except for the case $\left\langle S P_{\alpha \rightarrow 1}\left(G_{\mathrm{RG}}\right)\right\rangle$, see Figure 3(c).

It is quite remarkable to note that by substituting the average degree of (13) into (14) we get exactly the same expression of (11):

$$
\frac{\left\langle S P_{\alpha}\left(G_{\mathrm{RG}}\right)\right\rangle}{n} \approx \frac{1}{2}\langle d\rangle^{2} .
$$

So, in Figure 4 we plot again the average Stolarsky-Puebla indices reported in Figure 3 for RG graphs, but now normalized to $n$, as a function of $\langle d\rangle$ showing that all curves are now properly scaled. Also, in Figure 4, we show that (15) (orangedashed lines) indeed describes well the computational data (thick full curves) for $\langle d\rangle \geq 10$. We note that as well as for ER networks, here for RG graphs we do not observe the scaling of $\left\langle S P_{\alpha \rightarrow 1}\left(G_{\mathrm{RG}}\right)\right\rangle$.

\section{Discussion and conclusions}

We have introduced a degree-based variable topological index inspired on the Stolarsky mean, known as the generalization of the logarithmic mean. We named this new index as the Stolarsky-Puebla index $S P_{\alpha}(G)$, see (6). For given values of $\alpha$, 

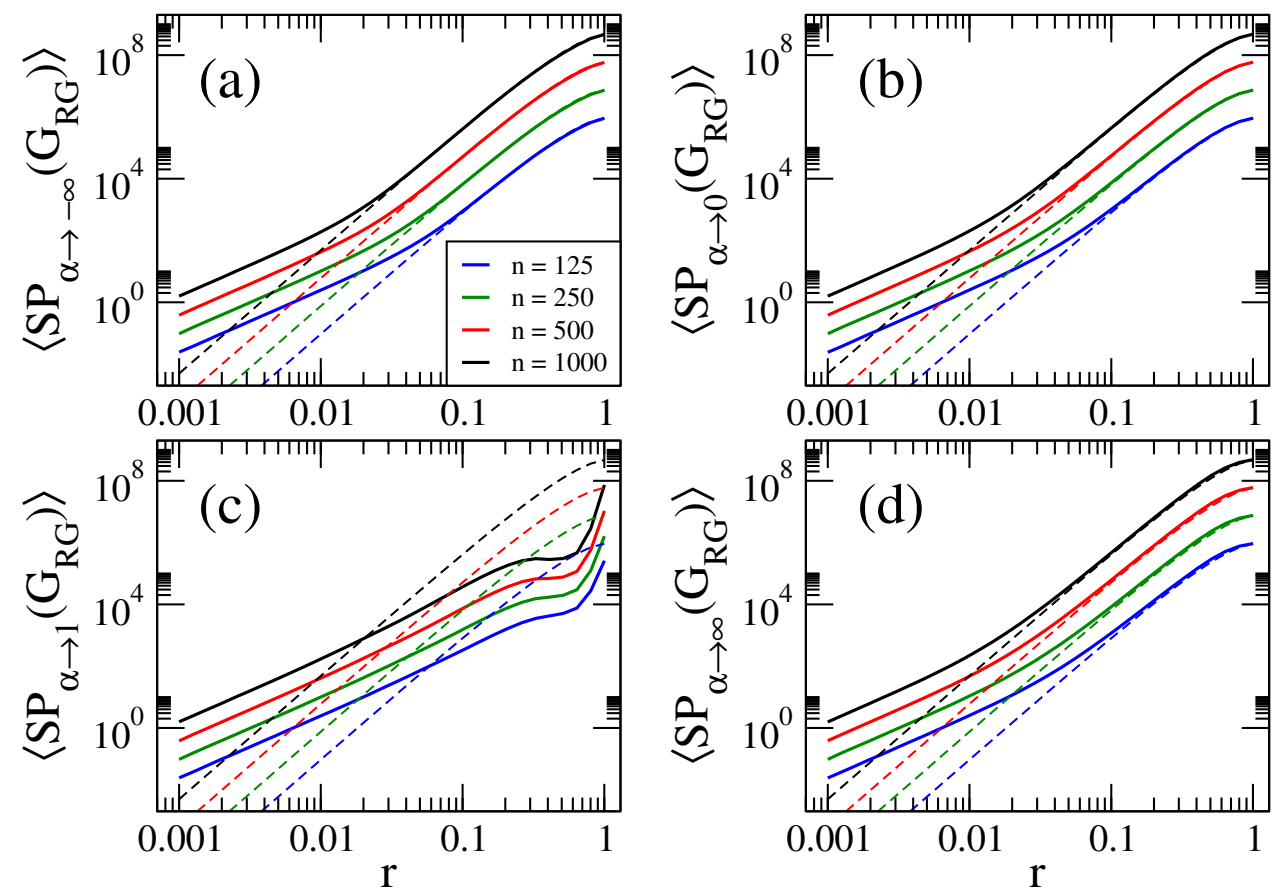

Figure 3: Average value of the Stolarsky-Puebla index $\left\langle S P_{\alpha}\left(G_{\mathrm{RG}}\right)\right\rangle$ as a function of the connection radius $r$ of random geometric graphs of size $n$. Here (a) $\alpha \rightarrow-\infty$, (b) $\alpha \rightarrow 0$, (c) $\alpha \rightarrow 1$, and (d) $\alpha \rightarrow \infty$. Dashed lines correspond to (14).
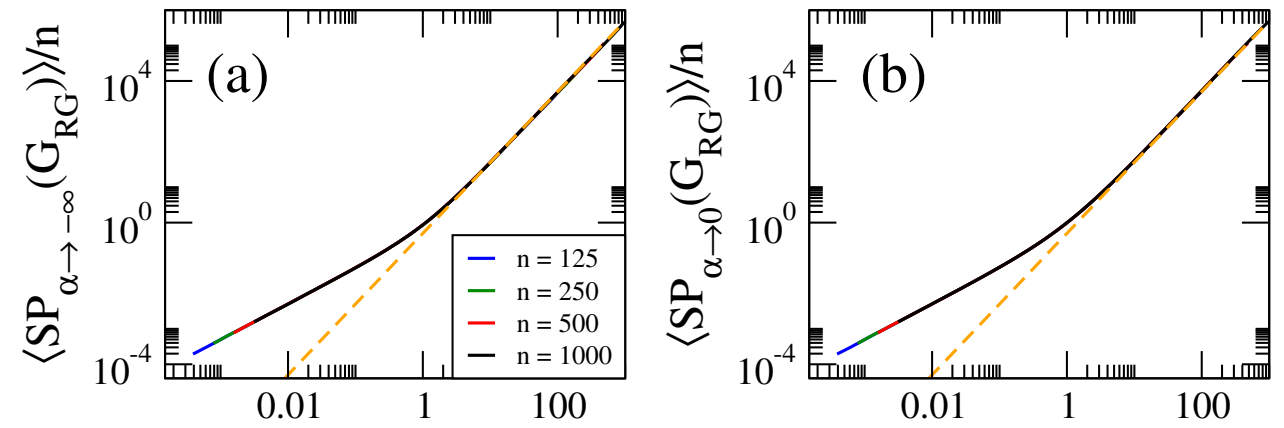

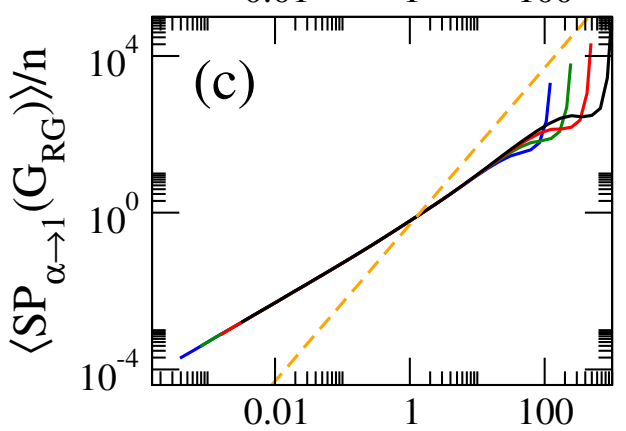

$\langle d\rangle$

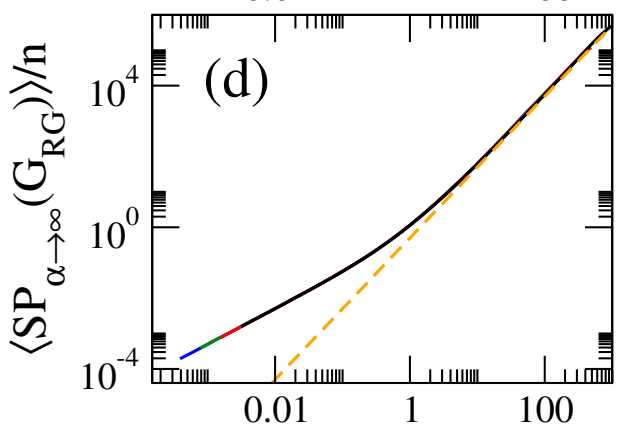

$\langle d\rangle$

Figure 4: Average value of the Stolarsky-Puebla index $\left\langle S P_{\alpha}\left(G_{\mathrm{GR}}\right)\right\rangle$, normalized to the network size $n$, as a function of the average degree $\langle d\rangle$ of random geometric graphs. Same curves as in Figure 3. Orange dashed lines are (15). The vertical magenta dashed lines indicate $\langle d\rangle=10$.

the Stolarsky-Puebla index is related to well-known topological indices, in particular it reproduces several mean Sombor indices $m S O_{\alpha}(G)$, see (7).

We want to add that the inequality of (4) can be straightforwardly used to state inequalities for the indices $S P_{\alpha}(G)$ and $m S O_{\alpha}(G)$, as well as for related indices:

$$
S P_{-1}(G)=m S O_{\alpha \rightarrow 0}(G) \leq S P_{\alpha \rightarrow 0}(G) \leq m S O_{1 / 3}(G) \leq S P_{2}(G)=m S O_{1}(G)
$$

or

$$
R^{-1}(G) \leq \operatorname{LogMean}(G) \leq m S O_{1 / 3}(G) \leq 2^{-1} M_{1}(G),
$$


which sets bounds for the logarithmic-mean topological index

$$
\operatorname{LogMean}(G)= \begin{cases}d_{u} & \text { if } d_{u}=d_{v}, \\ \frac{d_{u}-d_{v}}{\ln d_{u}-\ln d_{v}} & \text { otherwise }\end{cases}
$$

with respect to the reciprocal Randic index, the mean Sombor index with $\alpha=1 / 3$, and the first Zagreb index.

Since there are not many degree-based topological indices including logarithmic functions (as well-known exceptions we can mention the logarithms of the three multiplicative Zagreb indices [12] and the Adriatic indices [24,25]) we want to highlight the release of the logarithmic-mean topological index $\operatorname{LogMean}(G)$ of (18) as well as the identric-mean index

$$
\operatorname{idLogMean}(G)= \begin{cases}d_{u} & \text { if } d_{u}=d_{v}, \\ \frac{d_{u}-d_{v}}{d_{u} \ln d_{u}-d_{v} \ln d_{v}} & \text { otherwise }\end{cases}
$$

corresponding to $S P_{\alpha \rightarrow 0}(G)$ and $S P_{\alpha \rightarrow 1}(G)$, respectively.

We have also applied the Stolarsky-Puebla index $S P_{\alpha}(G)$ to Erdös-Rényi (ER) networks and random geometric (RG) graphs and within a statistical random matrix theory approach we demonstrated that $\left\langle S P_{\alpha}(G)\right\rangle$, normalized to the order of the network, scales with the corresponding average degree $\langle d\rangle$. However, it is fair to recognize that, for both random network models, $\left\langle S P_{\alpha \rightarrow 1}(G)\right\rangle=\langle\operatorname{idLogMean}(G)\rangle$ did not scale; so we believe that the identric-mean index deserves further investigation.

In addition, from (16) we are able to write an equivalent inequality but for the corresponding average values:

$$
\left\langle S P_{-1}(G)\right\rangle \leq\langle\operatorname{LogMean}(G)\rangle \leq\left\langle m S O_{1 / 3}(G)\right\rangle \leq\left\langle S P_{2}(G)\right\rangle .
$$

Indeed, we verified that (20) is satisfied for the both ER random networks and RG graphs (not shown here). Moreover, we computationally found that

$$
\langle\operatorname{idLogMean}(G)\rangle \leq\left\langle S P_{\alpha \neq 1}(G)\right\rangle,
$$

for the two random network models we study here (not explicitelly shown here but partially observed in Figs. 1 and 3). The equalities in (20) and (21) are attained when $p=1$ and $r=\sqrt{2}$, for ER random networks and RG graphs, respectively.

Finally, we want to recall that through a quantitative structure property relationship (QSPR) analysis it was shown [2] that $m S O_{\alpha \rightarrow \pm \infty}(G)$ are good predictors of the standard enthalpy of vaporization, the enthalpy of vaporization, and the heat of vaporization at $25^{\circ} \mathrm{C}$ of octane isomers. Furthermore, since $S P_{\alpha \rightarrow \pm \infty}(G)=m S O_{\alpha \rightarrow \pm \infty}(G)$, we can conclude that $S P_{\alpha \rightarrow \pm \infty}(G)$ correlate well with the aforementioned physicochemical properties of octane isomers.

In future works we plan to explore mathematical and computational properties of $S P_{\alpha}(G)$, as well as finding optimal bounds and new relationships with known topological indices.

\section{Acknowledgments}

The first author (J. A. Méndez-Bermúdez) acknowledges financial support from CONACyT (Grant No. A1-S-22706) and BUAP (Grant No. 100405811-VIEP2021). The research of the last author (José M. Sigarreta) was supported by a grant from Agencia Estatal de Investigación (PID2019-106433GBI00 /AEI/10.13039/501100011033), Spain.

\section{References}

[1] R. Aguilar-Sanchez, I. F. Herrera-Gonzalez, J. A. Mendez-Bermudez, J. M. Sigarreta, Computational properties of general indices on random networks, Symmetry 12 (2020) \#1341.

[2] J. A. Mendez-Bermudez, R. Aguilar-Sanchez, E. D. Molina, J. M. Rodriguez, Mean Sombor index, Preprint.

[3] R. Aguilar-Sanchez, J. A. Mendez-Bermudez, F. A. Rodrigues, J. M. Sigarreta, Topological versus spectral properties of random geometric graphs, Phys. Rev. E 102 (2020) \#042306.

[4] R. Aguilar-Sanchez, J. A. Mendez-Bermudez, J. M. Rodriguez, J. M. Sigarreta, Normalized Sombor indices as complexity measures of random networks, Entropy 23 (2021) \#976.

[5] P. S. Bullen, Handbook of Means and Their Inequalities, Kluwer, Dordrecht, 2003.

[6] B. C. Carlson, Some inequalities for hypergeometric functions, Proc. Amer. Math. Soc. 17 (1966) 32-39.

[7] J. Dall, M. Christensen, Random geometric graphs, Phys. Rev. E 66 (2002) \#016121.

[8] P. Erdös, A. Rényi, On random graphs, Publ. Math. (Debrecen) 6 (1959) 290-297.

[9] P. Erdös, A. Rényi, On the evolution of random graphs, Inst. Hung. Acad. Sci. 5 (1960) 17-61.

[10] P. Erdös, A. Rényi, On the strength of connectedness of a random graph, Acta Math. Hungar. 12 (1961) 261-267.

[11] E. Estrada, M. Sheerin, Random rectangular graphs, Phys Rev. E 91 (2015) \#042805.

[12] I. Gutman, Degree-based topological indices, Croat. Chem. Acta 86 (2013) 351-361.

[13] I. Gutman, B. Furtula, C. Elphick, Three new/old vertex-degree-based topological indices, MATCH Commun. Math. Comput. Chem. 72 (2014) $617-632$ 
[14] I. Gutman, N. Trinajstić, Graph theory and molecular orbitals. Total $\pi$-electron energy of alternant hydrocarbons, Chem. Phys. Lett. 17 (1972) $535-538$.

[15] V. R. Kulli, The (a,b)-KA indices of polycyclic aromatic hydrocarbons and benzenoid systems, Int. J. Math. Trends Tech. 65 (2019) 115-120.

[16] T. P. Lin, The power mean and the logarithmic mean, Amer. Math. Monthly 81 (1974) 879-883.

[17] C. T. Martinez-Martinez, J. A. Mendez-Bermudez, J. M. Rodriguez, J. M. Sigarreta, Computational and analytical studies of the Randić index in Erdös-Rényi models, Appl. Math. Comput. 377 (2020) \#125137.

[18] C. T. Martinez-Martinez, J. A. Mendez-Bermudez, J. M. Rodriguez, J. M. Sigarreta, Computational and analytical studies of the harmonic index in Erdös-Rényi models, MATCH Commun. Math. Comput. Chem. 85 (2021) 395-426.

[19] B. Ostle, H. L. Terwilliger, A comparison of two means, Proc. Montana Acad. Sci. 17 (1957) 69-70.

[20] M. Penrose, Random Geometric Graphs, Oxford University Press, Oxford, 2003.

[21] R. Solomonoff, A. Rapoport, Connectivity of random nets, Bull. Math. Biophys. 13 (1951) 107-117.

[22] K. B. Stolarsky, Generalizations of the logarithmic mean, Math. Mag. 48 (1975) 87-92.

[23] S. Sykora, Mathematical Means and Averages: Basic Properties, Volume 3, Stan's Library, Castano Primo, 2009.

[24] D. Vukičević, Bond additive modeling 2. Mathematical properties of max-min rodeg index, Croat. Chem. Acta 83 (2010) $261-273$.

[25] D. Vukičević, Bond additive modeling 5. Mathematical properties of the variable sum Exdeg index, Croat. Chem. Acta 84 (2011) 93-101. 